\title{
THE STABILITY OF THE CERVICAL SPINE FOLLOWING THE CONSERVATIVE TREATMENT OF FRACTURES AND FRACTURE-DISLOCATIONS
}

\author{
D. J. E. CHESHIRE, M.B., B.S., D.PHYS.MED. \\ Spinal Injuries Centre, Austin Hospital, Heidelberg, Victoria, Australia
}

THE early treatment of injuries of the cervical spine and spinal cord may be either conservative or operative. Differing views are well expressed in the following quotations:

'When the posterior ligament complex has been ruptured healing such as to restore the original strength does not occur. For example, it is common knowledge that after accurate reduction and prolonged immobilisation of dislocations of the cervical spine late redisplacement often occurs' (Holdsworth, I963).

'Evidence is presented to demonstrate by means of comparative studies of two groups of cervical spine fracture-dislocations, with or without cord or root complications, that treatment by operative fixation of the bones has a greater mortality, causes more undesirable symptoms and is less effective than treatment by skeletal traction without either internal fixation or fusion' (Munro, I96I).

'Operations are done because surgeons think spines may be unstable and thus it is safer to advise fusion. Perhaps this is safer for the Surgeon, but is such open surgery safe for the patient ?' (Beatson, I963).

The purpose of this paper is not to recapitulate the old arguments, but to attempt to answer one question which is fundamental to the controversy, and which has frequently been included amongst the indications for early surgery. This question is that of the stability of the unstable, or potentially unstable, cervical spine at the conclusion of conservative treatment. The literature has for too long contained such imprecise and emotive statements as 'it is COMMON KNOWLEDGE that after accurate reduction and prolonged immobilisation of dislocations of the cervical spine late redisplacement OFTEN occurs' (Holdsworth, I963) ... (author's capitals), and a definitive statement, derived from the study of an adequate series of cases, is overdue. An arithmetical analysis of late instability following conservative management is logically followed by an examination of the cases which showed late instability, and an attempt to determine whether these cases had any common pattern.

Definition. Stability is defined as 'the absence of any abnormal mobility between any pair of vertebrae, with or without pain or other clinical manifestations, when lateral X-rays of the cervical spine are taken in flexion and extension at the conclusion of the conservative treatment of a fracture or fracture-dislocation'.

Material. A personal series of 257 cases of acute injury to the cervical spine and spinal cord is presented. These patients were treated in the Spinal Injuries Centre for Victoria in the nine years from I July I959 to 30 June 1968.

In all cases injury to the cervical spine was associated with neurological signs, admission to the Centre was within I5 days of the injury (68 per cent. within 24 hours) and the treatment of the injury to the cervical spine was planned and executed by the author or the Assistant Director together with their orthopaedic and neurosurgical colleagues (Cheshire, I968). 
Classification of Cervical Injuries. There are many classifications of injuries of the cervical spine. This is not the place to debate their merits and demerits. Suffice it to say that in this paper the classification used is broadly based on that proposed by Holdsworth (1963), and also relates to the work of Roaf (I960) and Beatson (1963), whose important experimental work increased the general understanding of the mechanisms involved. However, and this point must remain to be argued later, it is felt that these authors oversimplify flexion-rotation injuries and Stringa's (1963) sub-group of 'anterior subluxations' is included.

\section{Classification and Incidence}

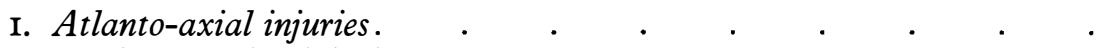

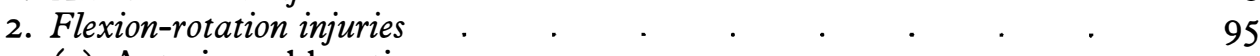

(a) Anterior subluxations . . . . . . . . . I9

(b) Flexion-rotation dislocations and fracture-dislocations with unilateral posterior intervertebral joint dislocation

(c) Flexion-rotation dislocations and fracture-dislocations with bilateral posterior intervertebral joint dislocations . . $\quad 35$

3. Extension-rotation injuries
(a) Extension disruption of normal spines and spines affected by cervical spondylosis

(b) Extension disruption of a spine affected by ankylosing spondylitis

(c) Extension injury of a spine affected by cervical spondylosis, but no bony injury or ligamentous disruption

(d) Complete Instability in Apparent Extension Injuries . . 6

4. Vertical compression injuries

5. No radiological abnormality on plain $X$-ray but permanent neurological deficit

(Pathology demonstrated at myelography and/or surgery-e.g. acute 6 cervical disc lesion)

6. Gunshot wound.

7. Normal X-ray. Cervical cord and/or root concussion $\quad . \quad$. $\quad$ I

Elimination of Inherently Stable Lesions. In this investigation, which is concerned with late instability following conservative management, groups $3(c), 5$, 6 and 7, totalling 33 patients, must be eliminated, leaving a total of 224 patients for analysis. The author is unable wholly to agree with Holdsworth's (I963) statement that vertical compression injuries are stable, because in his experience it is very difficult to differentiate the true vertical compression injury, in which, indeed, all the surrounding ligaments are intact, from that injury to the vertebral body which is caused by combined vertical-compression and flexion forces. In the latter there may be an initial radiological picture of a classical vertical-compression injury, but functional films at the conclusion of conservative treatment can reveal an instability which indicates rupture of the posterior ligament complex at the time of the injury. For this reason the author is becoming increasingly cautious with this lesion, and must include it amongst the groups in which late instability is, at least, a possibility.

Routine Treatment Practice. Injuries of the cervical spine and spinal cord are usually treated conservatively. The alignment of the neural canal is restored as rapidly as possible by conservative means and maintained until spontaneous healing 
has occurred. Prior to I96I, all flexion injuries were treated by the application of skull tongs, reduction, and then maintenance by skeletal traction. However, this method was not invariably successful, reduction was often too long delayed, and some patients had to come to open reduction using the posterior approach. Since Evans (I96I) revived the technique of manual manipulation of the cervical spine under general anaesthesia and relaxants, this technique has been the method of choice in early flexion-rotation injuries with unilateral and bilateral posterior intervertebral joint dislocations.

Whether the neural canal be realigned by traction or manipulation, skeletal traction is maintained for six to eight weeks. Thereafter the patient wears a cervical collar at all times, and, at about $\mathrm{I} 2$ weeks, functional films are taken in flexion and extension. If the spine is then unstable or there is evidence, clinical or radiological, of instability at a later date, the patient is considered for late spinal fusion.

Extension disruptions are treated by postural reduction in flexion, and the use of a cervical collar for 12 weeks, at the end of which time, again, functional films are taken.

Vertical compression injuries are treated by the application of skull tongs, and skeletal traction. The question of late instability is determined as above.

Cases in which Early Surgery was Performed. In the 224 patients with unstable or potentially unstable cervical spines, early surgery was performed I 2 times. These 12 patients divide into clearly differentiated groups.

I. In four patients open reduction by the posterior approach, combined in three patients with partial facetectomy, was performed following failure to reduce flexion-rotation dislocations with bilateral posterior intervertebral joint dislocations by skeletal traction. All four patients were, and remained, completely tetraplegic. Since the first manual manipulation of a cervical dislocation was performed in this Centre in November I96I no further posterior open reductions have been performed.

2. Three patients in group $3(d)$ of the classification were at first thought to have sustained the usual extension disruption in which the anterior longitudinal ligament is ruptured, but the posterior ligament complex remains intact. However, they later showed, by dislocating anteriorly when the neck was flexed, that they had sustained the comparatively rare Complete Instability in Apparent Extension Injuries. These patients were treated by early anterior cervical fusion, but three later patients with the same lesion were treated conservatively. This interesting lesion, which presents problems in both diagnosis and treatment, will be the subject of a separate publication.

3. Two young males sustained a bilateral fracture of the posterior arch of the axis in an extension injury, followed by forward dislocation of the body of $\mathrm{C}_{2}$ on C3. Initially both patients had major incomplete tetrapareses, but there was rapid neurological recovery. Both were treated initially by skeletal traction, but, because the reduction was inadequate and unstable, anterior spinal fusion was performed between $\mathrm{C}_{2}$ and $\mathrm{C}_{3}$. This is the lesion described by Cornish (1968).

4. Three other patients were also treated by early anterior spinal fusion. In summary, the reason for early surgery were, respectively:

(a) Failure of manipulative reduction of a unilateral posterior intervertebral joint dislocation due to the interposition of the fractured tip of the inferior facet in the dislocated joint. As the neurological signs were slight and improving, and the 
patient was a nursing mother, it was felt justifiable to perform early anterior spinal fusion to permit rapid discharge from hospital.

(b) A young man with advanced ankylosing spondylitis sustained a gross extension-disruption through the $\mathrm{C}_{5} / 6$ disc. The combination of an incomplete and recovering tetraparesis and the difficulties of maintaining conservative immobilisation in a man with a severe upper dorsal kyphosis led to the decision to fuse the spine.

(c) A young man sustained a double cervical lesion, a flexion-rotation fracturedislocation with bilateral posterior intervertebral joint dislocation at $\mathrm{C} 4 / 5$, and an anterior subluxation of $\mathrm{C} 6$ on $\mathrm{C}_{7}$. As this double lesion was accompanied by no more than an incomplete tetraparesis of Brown-Séquard type only one attempt at manual manipulation of the $\mathrm{C} 4 / 5$ dislocation was made. When this failed it was decided to proceed under the same anaesthetic to open reduction and anterior spinal fusion.

Late Instability following Conservative Treatment. Of the 224 patients with unstable or potentially unstable lesions of the cervical spine, 12 were treated by early spinal surgery. Thus 212 patients were treated conservatively, and of these I 53 were treated by skull tongs and skeletal traction (this group includes 40 patients in whom closed manual manipulation was performed), followed by a cervical collar, and 59 were treated in cervical collars alone. Twelve of these patients, or 5.4 per cent., demonstrated late instability.

Cases of Late Instability. The cases of late instability divide clearly into four groups:

I. Anterior subluxations.

2. Vertical compression injuries which later proved to be unstable in flexion due to rupture of the posterior ligament complex between the compressed vertebra and the vertebra above.

3. Unilateral posterior intervertebral joint dislocations in flexion-rotation dislocations and fracture-dislocations.

4. Bilateral posterior intervertebral joint dislocations in flexion-rotation dislocations and fracture-dislocations.

These groups will be discussed in turn:

I. Anterior Subluxations. Four of the cases of late instability were patients whose spinal lesion was an anterior subluxation.

Illustrative Case History. A 23-year-old female sustained an anterior subluxation between C6 and 7 (fig. I) with a slight wedge fracture of the body of $\mathrm{C}_{7}$. No abnormality was seen in the oblique films. Abnormal neurological signs were initially confined to the right upper limb, and consisted of a partial 7 th and 8th cervical nerve root lesion. The cervical spine was treated by application of skull tongs, and I6 lb. $\left(7 \cdot 2 \mathrm{~kg}\right.$.) traction in $20^{\circ}$ extension. Realignment of the cervical spine was successful and traction was maintained for six weeks. Neurologically, there was slow but progressive motor and sensory improvement in the right upper limb, but on the day following admission spasticity and hyper-reflexia was found in the legs. There were no motor or sensory abnormalities in the legs and bladder function was normal. When the tongs were removed at six weeks there was virtually complete recovery of all neurological functions. A cervical collar was worn from the sixth to the twelfth week, but lateral X-rays in flexion and extension (fig. 2, A and B) taken in the twelfth week showed subluxation in flexion, and this subluxation, with obvious posterior ligamentous instability and slight wedging of the body of $\mathrm{C}_{7}$, combined 
Fig. I-Anterior subluxation of $\mathrm{C}_{6}$ on $\mathrm{C}_{7}$, with slight wedge fracture of the body of $\mathrm{C}_{7}$.

Fig. 2-A and B. Functional films show instability of $\mathrm{C} 6$ on $\mathrm{C}_{7}$ in flexion.

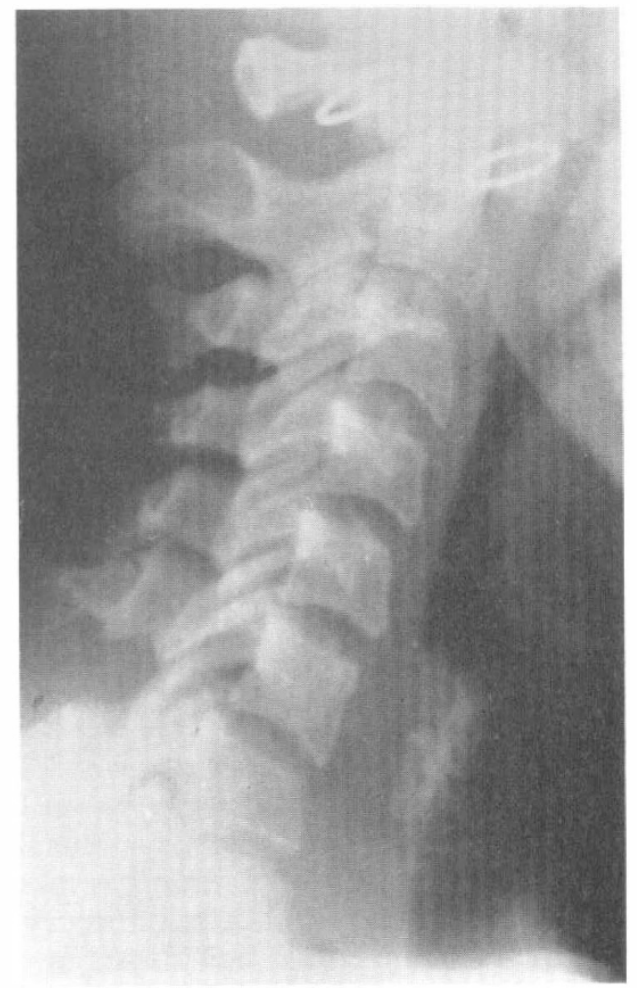

FIG. I
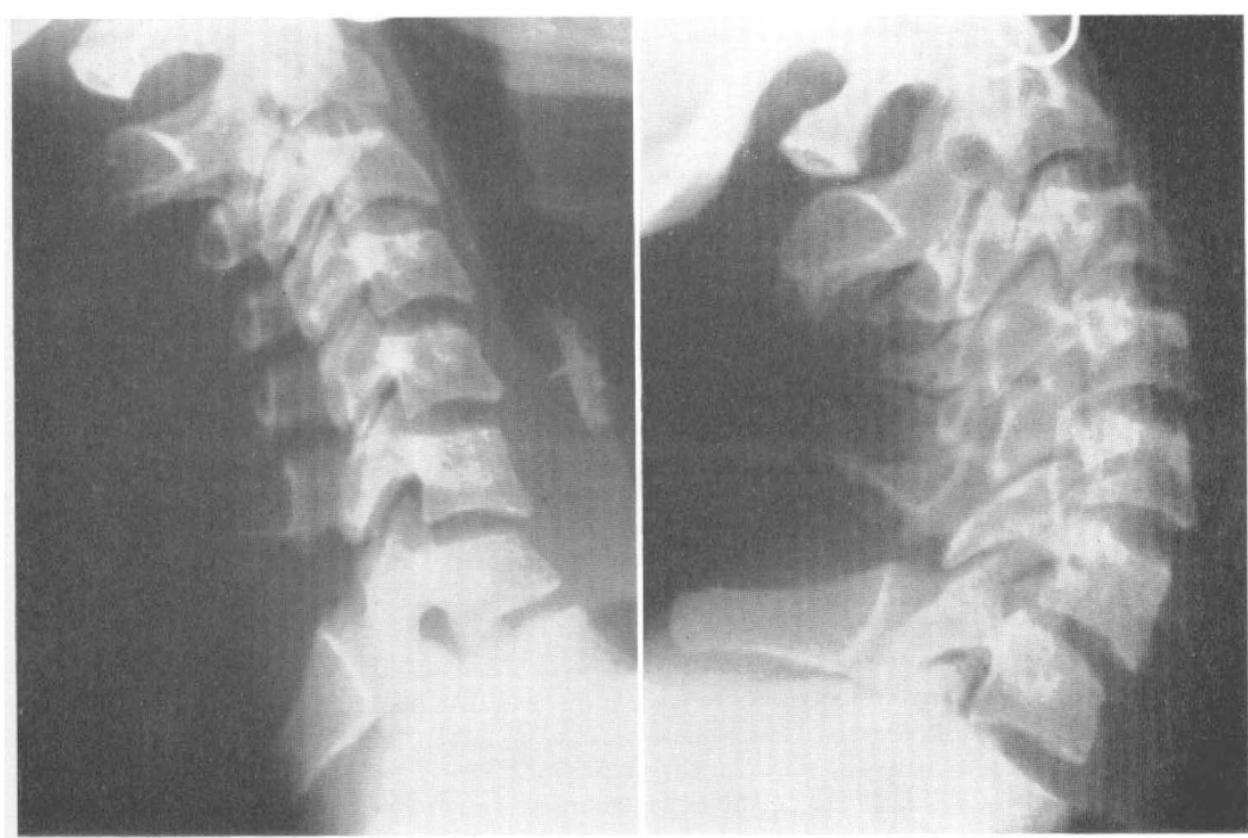
with the excellent neurological result, led to a decision to perform anterior spinal fusion at C6/7.

In presenting a classification of cervical injuries the opinion was expressed that it is an over simplification to recognise only two stages in flexion-rotation injuries. That 4 of the I 2 cases of late instability were anterior subluxations reinforces the opinion that Stringa ( 1963 ) was correct to include this entity in his classification of cervical injuries. Stringa states: 'trauma is fundamentally in flexion, and the fundamental result is the anterior subluxation of the body; but this is most frequently accompanied by a wedge fracture of a body underneath'. Even if the trauma be fundamentally due to a flexion force, the fact that, in the present series, 4 out of 19 cases ( 2 I per cent.) of anterior subluxation demonstrated late instability suggests that there is also a rotary element. Roaf's (I960) experimental work showed that there must be a rotation force before ligamentous tearing occurs, and leads to the proposition that this lesion should be recognised as an intermediate stage between a pure flexion injury which produces a wedge fracture of a vertebral body, with all ligaments remaining intact, and a flexion-rotation injury with unilateral posterior intervertebral joint dislocation, which results when the posterior ligament complex and the peri-articular ligaments of one posterior intervertebral joint are ruptured.

2. Vertical Compression Injuries. Three patients sustained vertical compression fractures of a vertebral body, but lateral X-rays taken in flexion at 12 weeks showed instability and anterior subluxation between the compressed vertebra and the vertebra above. This finding is taken as evidence that the original lesion must have been a vertical compression injury sustained whilst the neck was flexed, and that the flexion element was missed in the early examination.

Illustrative Case Histories. A I4-year-old male dived into shallow water and sustained a vertical compression fracture of $\mathrm{C}_{5}$ with posterior intrusion into the neural canal (fig. 3 ). He presented with a major, but incomplete, tetraparesis of Brown-Séquard pattern. $\mathrm{He}$ was treated by six weeks' skeletal traction in the neutral plane, followed by six weeks in a cervical collar. At the twelfth week lateral X-rays in flexion and extension (fig. 4, A and B) demonstrated considerable instability and anterior subluxation of $\mathrm{C}_{4}$ on $\mathrm{C}_{5}$. The patient was making a good, though incomplete neurological recovery, and it was decided to perform a spinal fusion. At that time (March 1960) the operation performed was a posterior exposure of the spine, which demonstrated the rupture of the posterior ligament complex between $\mathrm{C}_{4}$ and $\mathrm{C}_{5}$ very clearly, followed by wiring of the spinous processes and an on-lay bone graft.

The second case is included because it is felt that there is an important lesson to be learned from it. For many years the terms 'burst fracture', and 'tear-drop fracture' have been used as alternatives and synonyms for the lesion now usually referred to as a vertical compression fracture. This case is presented because it demonstrates that the classical 'tear-drop' appearance is that of a vertical compression and flexion injury and not of a pure vertical compression injury.

An I8-year-old female sustained a classical 'tear-drop' fracture of C6 (fig. 5). The neck was treated by six weeks in traction and six weeks in a collar, and the patient made a virtually complete neurological recovery. However, functional films at I2 weeks show gross instability and anterior subluxation at $\mathrm{C}_{5} / 6$ (fig. 6, $\mathrm{A}$ and $\mathrm{B}$ ). It is felt that if one reviews these X-rays with the wisdom of hindsight it is not difficult to visualise a major flexion force as being the cause of the separation of the 'tear-drop' fragment. 
Fig. 3-Vertical compression injury of $\mathrm{C}_{5}$.

Fig. 4-A and B. Functional films show instability of $\mathrm{C}_{4}$ on $\mathrm{C}_{5}$ in flexion.

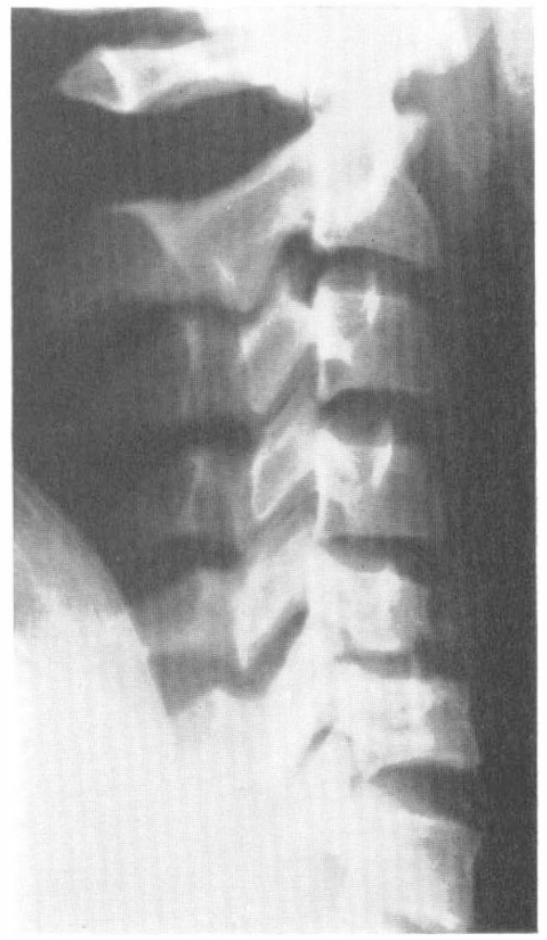

FIG. 3

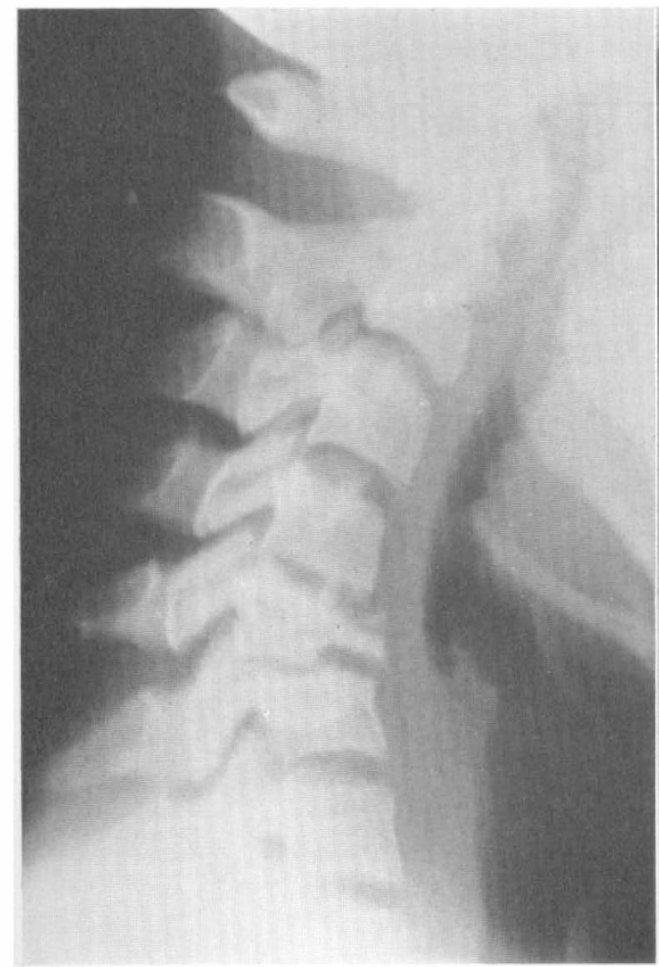

A

FIG. 4 


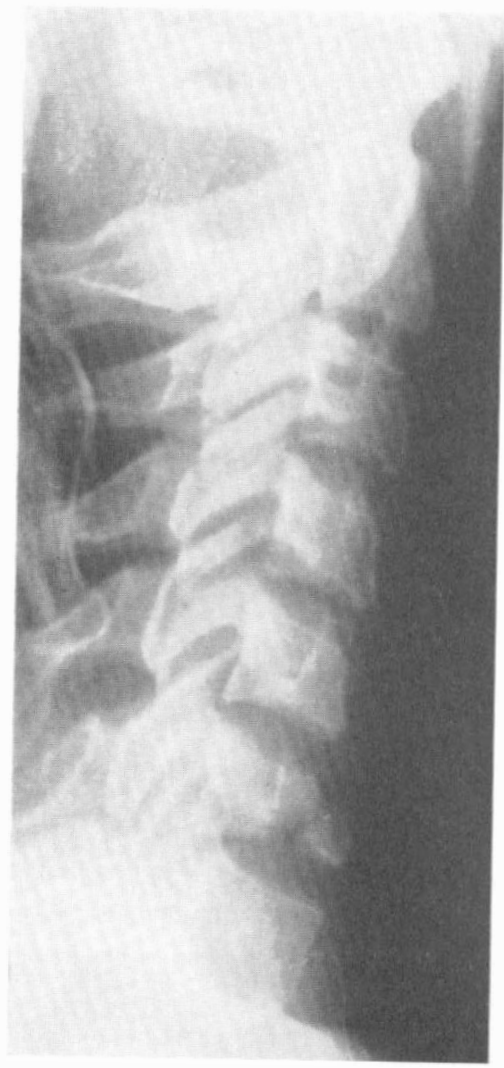

Fig. 5-'Tear-drop' fracture of $\mathrm{C} 6$.

Fig. 6-A and B. Functional films show instability of $\mathrm{C}_{5}$ on C6 in flexion.

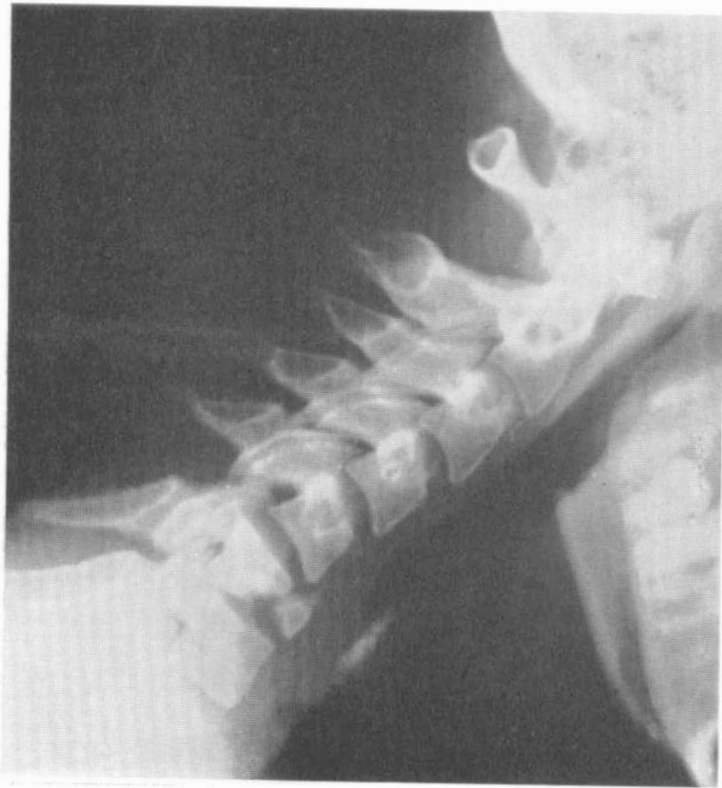

A

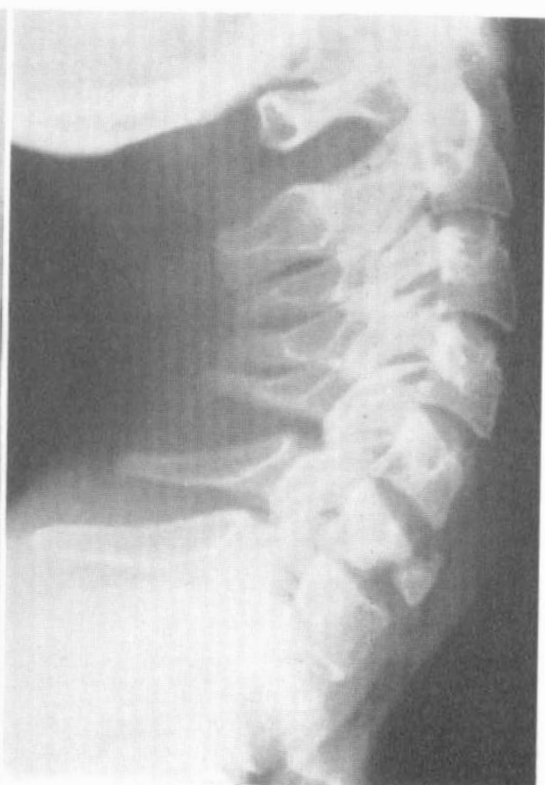

B 
It is suggested that such 'tear-drop' fractures should be treated with suspicion, and may perhaps be considered for early spinal fusion, especially if the patient has minimal neurological signs. A perusal of the literature on this lesion is rewarding. In publications to which previous reference has been made, Holdsworth (1963) and Beatson (1963), under the discussion and specific label of 'burst' fractures, present X-rays of spines which show classical 'tear-drop' fractures. On the other hand, Schneider and Kahn stated in 1956 that 'the acute flexion or tear-drop fracture dislocation is characterised by separation and downward and forward displacement of the antero-inferior margin of the involved vertebral body. The postero-inferior margin is displaced posteriorly into the spinal canal.' The author therefore claims no originality in his contention that it is vital to differentiate between the true vertical compression fracture, which, as Holdsworth (1963) states, is a stable injury, and the 'acute flexion or tear-drop fracture-dislocation' which Schneider described in 1963 as a very unstable lesion. The case quoted demonstrates this instability.

3. Flexion-rotation Dislocations, with Unilateral Posterior Intervertebral foint Dislocation. There were three patients in this group. All had very incomplete cord signs and they were treated by manual manipulation of the neck followed by skeletal traction for six weeks. All three re-dislocated between two and three weeks later whilst wearing cervical collars, and because, in all three cases, complete neurological recovery had taken place, it was decided to perform a spinal fusion.

4. Flexion-rotation Dislocations with Bilateral Posterior Intervertebral foint Dislocations. Two patients with this lesion re-dislocated, one about a month after the accident, whilst critically ill with respiratory insufficiency due to pulmonary consolidation and collapse. Pre-occupation with the respiratory problems resulted in inadequate management of the cervical spine, which had been reduced by manual manipulation, and was being maintained by skeletal traction. At the time the re-dislocation was noticed the patient's general condition precluded active treatment of the neck, and later, when the patient's condition improved, there was radiological evidence of bony union in the dislocated position. Because the patient's neurological condition remained unchanged (complete tetraplegia below $\mathrm{C}_{5}$ ) and there were no neck symptoms, no active treatment was performed and the neurological condition remains unchanged seven years later.

In the second patient, whose skeletal injury was at $\mathrm{C}_{5} / 6$ and who was completely tetraplegic below C6, the early management was also by manual manipulation, followed by six weeks' traction, then six weeks in a collar. Functional films at 12 weeks were satisfactory but, about a month later, the patient developed bilateral root pain in the $\mathrm{C}_{5}$ dermatome, and X-rays showed re-dislocation. Because of the persistent pain and the possible functional consequences, anterior reduction and fusion was performed.

\section{CONCLUSIONS}

From a total series of 257 patients with acute injuries of the cervical spine and spinal cord and/or nerve roots, 2 I 2 patients were treated conservatively by realignment of the neural canal by skeletal traction or manual manipulation, followed by the maintenance of traction for six to eight weeks, and then by the wearing of a cervical collar until the twelfth week. Of these 212 patients, Io were demonstrated to be unstable when lateral X-rays were taken in flexion and extension at the twelfth week, and two were demonstrated to have re-displaced but to have stable lesions 
with bony union in the position of displacement. An unsatisfactory result is therefore judged to have occurred in 12 out of $2 \mathrm{I} 2$ patients ( 5.4 per cent.).

Analysis of the cases of late instability in terms of the individual lesion types demonstrates:

(A) Atlanto-axial injuries-

8 cases-none unstable

(B) Flexion-rotation injuries-

(i) Anterior subluxations:

I9 cases -4 unstable (2I per cent.)

(ii) Flexion-rotation dislocations and fracture-dislocations with unilateral posterior intervertebral joint dislocation: 4I cases-3 unstable $(7 \cdot 3$ per cent.)

(iii) Flexion-rotation dislocations and fracture-dislocations with bilateral posterior intervertebral joint dislocation: 35 cases -2 unstable ( 5.7 per cent.)

(C) Extension-rotation injuries, with anterior ligament disruption (i.e. classification groups $3(a)$ and $(b))$

52 cases-none unstable

(D) Vertical compression injuries, including those with a flexion element63 cases-3 unstable ( 4.8 per cent.)

To report a late instability rate of $5 \cdot 4$ per cent. is of some interest, but when further analysis shows that the rate of instability in so large a group as the extensiondisruptions ( 24.5 per cent. of all cases treated conservatively) is nil, it is apparent that only an individual analysis of the separate lesion groups is truly meaningful. If the extension disruptions are eliminated from the reported series of 'unstable or potentially unstable' lesions, the remaining I60 patients showed a late instability rate of 7.5 per cent.

So far as late instability is concerned, it is felt that the individual analyses permit a case to be made for the conservative management of all groups of unstable or potentially unstable lesions of the cervical spine except the anterior subluxations.

It is imperative to seek the reason why anterior subluxations should show a 2I per cent. late instability rate when flexion-rotation dislocations with unilateral and bilateral posterior intervertebral joint dislocations showed much lower rates of 7.3 per cent. and 5.7 per cent. respectively. It is postulated that these lesions are stages in the same mechanism, but posterior intervertebral joint dislocations must have at least as much, and probably more, posterior ligamentous tearing than anterior subluxations. It is felt, therefore, that the answer probably lies in the adequacy of the conservative management of the different lesions, rather than in either the lesions themselves or the essential treatment techniques. Fifteen of the I9 anterior subluxations were associated with minor and rapidly recovering neurological manifestations and the patients were consequently much more mobile. Despite the quotation from Holdsworth (1963) with which this paper opened, it is felt that if a torn ligamentous system be immobilised well enough, and for long enough, satisfactory healing will result. Immobilisation by adequate conservative techniques must be granted to be within the competence of any good Spinal Injuries Centre; in fact, the conservative methods which Sir Ludwig Guttmann pioneered are based on the assertion that a Spinal Injuries Centre is a special Unit in which the techniques of spinal nursing are perfected to the state where unstable and 
potentially unstable skeletal injuries may be treated conservatively without risk of further damage to the spinal cord, so permitting the patient to avoid early spinal surgery. Conservative techniques are certainly easier to practise in the tetraplegic than in a patient with a minimal neurological lesion who is much more actively mobile, and influenced by his opportunity to observe more minor and transient cervical cord lesions than are seen in many other Centres, and by his knowledge of the greater difficulty in maintaining adequate conservative immobilisation in these patients, the author finds a late instability rate of 2 I per cent. in anterior subluxations disturbingly high, and is presently re-appraising the possibility of opting for early anterior spinal fusion in these cases.

It is well appreciated that, in a patient with an injury to the cervical spine and spinal cord, it is impossible to consider the treatment of the spine as a separate entity without reference to the correct treatment of the injured spinal cord, but the present investigation is directed towards the single question of spinal stability, leaving the functional end-results of the cord injury to be analysed in another paper. It is also appreciated that neither spinal stability nor functional end-results can accurately be assessed without reference to the death rate in the particular series being analysed. This must also await a further paper, but inferential evidence is given in a previous publication (Cheshire, I968).

\section{SUMMARY}

A series of 257 cases of acute injury to the cervical spine and spinal cord is analysed to determine the incidence of late instability following conservative management.

When those cases were eliminated in which early surgery was performed, and those in which there was no possibility of late instability, there remained 160 cases which were treated conservatively. In these cases there was a 7.5 per cent. incidence of late instability.

The series is analysed in relation to each mechanico-anatomical lesion of the spine. In all the main groups, except one, the late instability rate was between 4.8 and 7.3 per cent. The exception was the anterior subluxations, in which four out of nineteen cases (2I per cent.) showed late instability. The mechanism of this lesion and its treatment are discussed.

The author wishes to thank his orthopaedic and neurosurgical colleagues, and the Assistant Director of the Centre, Dr. D. C. Burke, who participated in the treatment of the patients under review.

\section{REFERENCES}

Beatson, T. R. (1963). F. Bone Ft Surg. 45-B, 2 I.

Cheshire, D. J. E. (I968). Int. F. Paraplegia, 6, 59.

CoRNish B. L. (1968). F. Bone ft Surg. 50-B, 3 I.

Evans, D. K. (I961). F. Bone ft Surg. 43-B, 552.

HoldsworTh, F. W. (1963). F. Bone ft Surg. 45-B, 6.

Munro, D. (1961). New Engl. F. Med. 264, 573.

RoAf, R. (I960). F. Bone ft Surg. 42-B, 8I0.

SCHNEIDER, R. C. (1963). Proceedings of a Symposium on Spinal Injuries, ed. Harris, P., pp. I58-159. Royal College of Surgeons of Edinburgh.

SCHNEIDER, R. C. \& KAHN, E. A. (1956). F. Bone ft Surg. 38-A, 985.

Stringa, G. (1963). Proceedings of the IXth Congress of the International Society of Orthopaedic Surgery and Traumatology, pp. 69-97. Brussels: Imprimerie des Sciences. 